\title{
Perspective on experimental evaluation of adsorption energies at solid/liquid interfaces
}

\author{
Aleksandar R. Zeradjanin ${ }^{1} \cdot$ loannis Spanos ${ }^{1} \cdot$ Justus Masa $^{1} \cdot$ Michael Rohwerder $^{2} \cdot$ Robert Schlögl $^{1,3}$
}

Received: 8 April 2020 / Revised: 28 August 2020 / Accepted: 30 August 2020 / Published online: 10 September 2020

(C) The Author(s) 2020

\begin{abstract}
Almost 15 years ago, first papers appeared, in which the density functional theory (DFT) was used to predict activity trends of electrocatalytic reactions. That was a major contribution of computational chemistry in building the theory of electrocatalysis. The possibility of computational electrocatalyst design had a massive impact on the way of thinking in modern electrocatalysis. At the same time, substantial criticism towards popular DFT models was developed during the years, due to the oversimplified view on electrified interfaces. Having this in mind, this work proposes an experimental methodology for quantitative description of adsorption energies at solid/liquid interfaces based on the Kelvin probe technique. The introduced approach already gives valuable trends in adsorption energies while in the future should evolve into an additional source of robust values that could complement existing DFT results. The pillars of the new methodology are established and verified experimentally with very promising initial results.
\end{abstract}

Keywords Electrocatalysis $\cdot$ Adsorption energy $\cdot$ Kelvin probe $\cdot$ Interface $\cdot$ Activity trends $\cdot$ Work function

\section{Introduction}

This work belongs to the collection of papers dedicated to the 70th birthday of Prof. Jose Zagal. Our roads crossed for the first time more than 10 years ago. About the time we met, he was one of the speakers/participants at a workshop in Alicante (Spain) dedicated to electrocatalysis, which gathered the most important experts in the world at that time. He exhibited the remarkable level of in-depth knowledge and the understanding of electrochemistry, contributing very insightful comments/questions for practically every single talk at the workshop. We found out that Jose was at Case Western University where he worked with Ernst B. Yeager, founder of the first Electrochemistry Center in the USA, and probably

Aleksandar R. Zeradjanin

aleksandar.zeradjanin@cec.mpg.de

1 Max Planck Institute for Chemical Energy Conversion, Stiftstrasse 34-36, 45470 Mulheim an der Ruhr, Germany

2 Department of Interface Chemistry and Surface Engineering, Max-Planck-Institut für Eisenforschung, $\mathrm{GmbH}$, Max-Planck-Strasse 1, 40237 Dusseldorf, Germany

3 Fritz-Haber-Institut der Max-Planck Gesellschaft, Faradayweg 4-6, 14195 Berlin, Germany one of the leading electrochemists of the Western World, especially in the field of electrocatalysis [1]. Jose's research interests are relatively broad; however, he is recognized as a prominent figure in the field of electrocatalysis of oxygen reduction reaction (ORR) on macrocyclic molecules, focusing on tuning the catalytic activity of macrocycles by rational design of ligands, introducing new catalytic descriptors, as well as by establishing of various free energy relations [2]. In the special issue of this journal published in 2011, together with John Appleby, Jose co-authored an excellent review with the title "Free energy relations in electrochemistry, a history that started in 1935" [3]. This paper gives a very useful analysis of the main models used to predict the rates of electrochemical reactions and in part was inspiration for this work.

For almost 100 years, one of the most investigated phenomena in electrochemistry is the origins of electrocatalytic activity [4]. With time, understanding the reaction pathways and mechanisms based on parameters of electrode kinetics was upgraded with underlying co-relations between the nature of the electrode material, the structure of the electrode/ electrolyte interface, and the reaction rate $[5,6]$. After the "bridge" between the exact kinetic approach and the correlative descriptive approach was established [7, 8], further development was in the direction of identifying the key intermediate(s) [9] and then searching for materials with an optimal 
catalyst-intermediate bonding [10], giving mainly an emphasis on the thermodynamics of adsorption [11]. An optimal catalyst-intermediate bonding ("not too strong, not too weak") is recognized as the key prerequisite for a high/performance catalyst, known also in heterogeneous catalysis as the Sabatier principle [12]. This rather qualitative rule became a widely accepted activity criteria in electrocatalysis [13] and was also used to explain the activity of complex multielectron reactions [14]. Somewhere in 2004/2005, a group around Norskov proposed the density functional theory (DFT) as a quantitative frame for describing the electrocatalytic activity. Namely, they first introduced computational activity trends for oxygen reduction reaction (ORR) on metals and afterwards, they correlated experimentally well-established activities for the hydrogen evolution reaction (HER) with computationally derived values of proton adsorption energies $[15,16]$. That was a breakthrough of computational chemistry in the field of electrochemical reactions. The work of Norskov and followers marked a new era in electrocatalysis. It not only gave the possibility to complement experimental investigations, but it also opened a perspective to predict new electrocatalytically active materials. Finally, the approach of Norskov attempted to solidify the Sabatier principle as the main guiding principle in electrocatalysis research, which appears to have formed a broad perception amongst researchers that everything essentially important about the nature of the electrocatalytic activity has already been discovered. It seemed that the only thing remaining was to search for new materials with advanced properties. Afterwards, there was a lot of, mostly informal, criticism of the work of Norskov, usually in a very general narrative, with only few authors adducing comprehensive analysis of well-reasoned arguments to support the criticisms $[17,18]$. The argumentation relates to the impact of phenomena that are practically neglected or not addressed at all in the conventional computational models: (1) complexity of electrocatalyst surface (polycrystalline materials can have catalytic properties significantly different from any of the singlecrystal constituents), (2) impact of interfacial effects (electric field, oriented water dipoles in water bilayer, noncovalent interactions between water molecules and intermediate species), (3) impact of the electrolyte ( $\mathrm{pH}$ value, ions in the double layer including impurities), and (4) impact of the regime (far away from equilibrium the shape of potential energy curve and the activation barrier can change, including symmetry factor) [17-22].

Considering that the only existing systematic experimental dataset on adsorption energies, (determined by Krishtalik from electrochemical measurements as operative adsorption heat that includes all interactions in the double layer related to the formation of the hydrogen evolution intermediate) was subject to controversy [18], of major importance would be to develop experimental methodology, complementary to the computational one, that could overcome at least some of the limitations existing in conventional computational models. We initiated research in this direction earlier [21]. Despite the fact that the approach was intuitive and relied on some existing experimental correlations, it lacked important clarifications, including a rigid theoretical background [21]. Therefore, in this work, the scanning Kelvin probe (SKP), together with appropriate theoretical framework [23], is utilized at the interface of polycrystalline metals and water to estimate the energy of metalhydrogen $(\mathrm{M}-\mathrm{H})$ bond formation, an essential parameter in understanding the kinetics, mechanism, and activity trends of the hydrogen evolution reaction (HER).

\section{Note on the methodology}

First, it is essential, according to our current understanding of the reaction mechanism, to visualize the interfacial structural changes during the proton adsorption process on molecular level. Establishing a visual heuristic model can help us to identify more easily which phenomena and corresponding physicochemical quantities are important in the formation of $\mathrm{M}-\mathrm{H}$ bonds at open circuit conditions as well as how these quantities are expected to change with the applied potential [24]. Further, quantities relevant for the $\mathrm{M}-\mathrm{H}$ bond formation should be brought into relation with quantities used in describing the concept of the absolute electrode potential [25] including the IUPAC (International Union of Pure and Applied Chemistry) recommendation note on interfaces of conductive phases [26], which are at the very core of the working principle of SKP [23].

\section{Evolution of interfacial structure during proton adsorption}

The formation of a M-H bond is achieved through one of the simplest electrochemical elementary reactions known as Volmer reaction, where a proton from the electrolyte and an electron from the Fermi level of the metal interact at the inner Helmholtz plane (IHP) of the double layer forming atomic hydrogen adsorbed at the metal/electrolyte electrified interface [21]. Despite hydrogen adsorption being possible also at potentials more positive than potential of zero charge $\left(E_{\mathrm{pzc}}-\right.$ potential at which total surface charge is equal to 0 and where the probability of the orientation of interfacial water molecules both with oxygen and with hydrogen towards the metal surface is approximately the same [27]), analysis will be based on potential region more negative than $E_{\mathrm{pzc}}$. It should be taken into account that the surface water layers are disordered and that the observed interfaces are dynamic. This means that water molecule orientation is not in a static state, but rather more probable in a dynamic state involving various motions and configurations. In other words, when it is stated that at potentials more negative than $E_{\mathrm{pzc}}$ orientation of water molecule is 
with hydrogen towards the surface, this indicates increased probability of orientation for a molecule that is in a dynamic pattern of constant motion including rotations, vibrations, and rearranging of hydrogen binding network. Therefore, the content of Fig. 1 can be understood conditionally as snapshots in particular instances. Although perceived as simple, Volmer's step is in fact a complex process composed of several steps [24]. Namely, with applying a more negative potential than the potential of zero charge, the surface charge becomes negative. Consequently, there is an intuitive expectation that interfacial water molecules will be oriented with hydrogen towards the metallic surface, including gradual rearrangement of noncovalent hydrogen bonding as shown in Fig. 1a. This process is expected to be intensified as the electrode potential approaches the reversible potential for HER. Many authors support the notion that significant water reorientation is possible, including data in the well-known work of Iwasita [28]; however, in a very comprehensive review, Thiel et al. claimed that adsorbed water exhibits a multitude of geometries to accommodate hydrogen bonding network [29], which would formally make our illustrations of water orientation in Fig. 1 misleading or at least not accurate. In other words, at potentials more negative than $E_{\mathrm{pzc}}$ orientation is with $\mathrm{O}$ orientated to the surface, some molecules may have the opposite orientation and favor $\mathrm{H}$ adsorption. Nevertheless, as previously stated, our illustration is a heuristic model which does not necessarily represents reality but rather points out on the importance of underlying phenomena, in this case metal-water interaction that is a process parallel to the adsorption of a proton. Further, a proton will be transported from the outer to the inner Helmholtz plane, bringing positive charge closer to the negatively charged surface (Fig. 1b). This is formally represented by a shift of the proton together with the solvation shell (Fig. 1b and c), although in reality, the proton is transported probably by the Grotthuss mechanism, jumping from one solvation shell into another. The proton solvation shell at IHP is most probably getting deformed, due to the strong interfacial electric field, having as a consequence that the positive charge of the proton is more exposed to the negatively charged metallic surface in comparison with the solvation shell at the outer Helmholtz plane (OHP), as shown in Fig. 1c. In the same scheme (Fig. 1c), it is shown that the interfacial water layer is getting reorganized,
Fig. 1 Interfacial structural changes during proton adsorption at the metal/electrolyte boundary including the following: a the reorganization of the interfacial water layer under the influence of an electric field in the double layer given as change in entropy $\left(\Delta S_{\text {reor }}\right)$, b the work to transfer a proton $\left(W_{\mathrm{H}_{+}}\right)$from the outer Helmholtz (OHP) to the inner Helmholtz plane (IHP), c free energy of solvation shell deformation $\left(\Delta G_{\text {ssd }}\right)$ at the inner Helmholtz plane coupled with further reorganization of the interfacial water layer, and d electron transfer from the Fermi level of the metal towards the solvated proton with a partially deformed solvation shell exposing the positive charge. Color code: metal surface - gray, oxygen - blue, hydrogen from water-red, proton and adsorbed proton - pale red. Space was left intentionally between the first and the second interfacial water layer, for the purpose of clarity
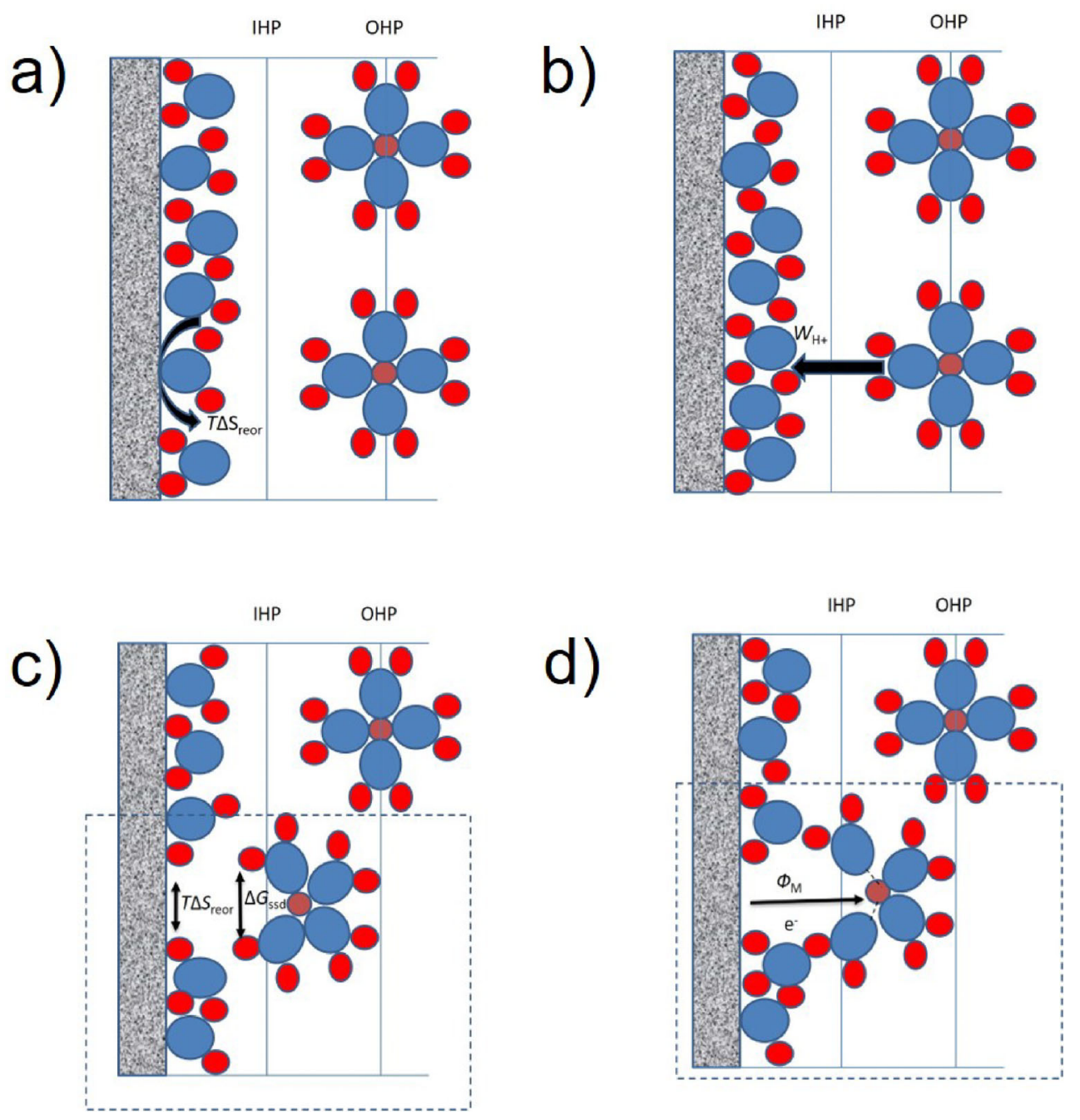
increasing the local density of the surface water layer, facilitating free space at the electrode surface for the $\mathrm{M}-\mathrm{H}$ bond to be established. Important to have in mind is that the solvation shell around the proton is in constant vibration by stretching the bonds between the proton and the surrounding water molecules. In the instance when (1) the energy of the electron at the Fermi level of the metal is high enough with respect to the redox potential of the electrolyte, (2) the solvation shell of the proton is properly oriented and deformed enough to expose the positive charge of the proton, and (3) the bonds between the proton and surrounding water molecules are sufficiently stretched additionally exposing the positive charge of the proton. Under these conditions, the probability that the electron will interact with a proton at the IHP becomes very high (Fig. 1d).

Most of the mentioned phenomena (water reorientation favoring intensified orientation of the hydrogen atoms towards the metal surface, approach of proton to the electrode surface including deformation of solvation sphere and exposure of positive charge, and finally $\mathrm{M}-\mathrm{H}$ bond formation) have a common denominator and that is the continuous change in the work function of the metal $\left(\Phi_{\mathrm{m}}\right)$.

\section{Link between work function, adsorption energy, and the measuring principle of Kelvin probe}

Figure 2 is a graphic illustration of the metal-electrolyte interface. The work function of the metal is the energy required to extract an electron from the Fermi level $\left(E_{\mathrm{F}}\right)$ of the metal and place it just outside of the metal surface where image forces can be neglected. It depends on the chemical potential of the electrons $(\mu-$ work to transfer the electron from infinity into the Fermi level of the uncharged metal) and the surface potential ( $\chi$ - electrostatic work to transport the electron through the dipole layer of the surface or in other words the potential drop between just inside the bulk and just outside of it). The surface potential appears because an electron cloud hovers over the surface, causing a potential difference with respect to the bulk. It is significantly affected by the crystal face orientation, the coordination number, and the step density. Evidently, the work function is simultaneously a bulk and surface property. This makes it quite unique amongst many other material properties and potentially interesting for understanding the electrocatalytic properties of the materials. In the general case, the metal could be charged and then an additional work component is required to transfer the electron from just outside the surface $\left(E_{\mathrm{vsc}^{*}}\right)$ to a position infinitely far away $\left(E_{\mathrm{vsc}}\right)$, known as Volta or outer potential $(\psi)$, which is equivalent to the potential drop from infinity to a position just outside the surface. The sum of the surface and the Volta potential is the inner or Galvani potential $(\varphi)$. Galvani potential represents the total electrostatic potential of one phase and together with the chemical potential constitutes the electrochemical potential $\left(\mu_{\text {elchem }}\right)$. Practically, all the mentioned quantities, depicted in Fig. 2, are applicable to a metal and to an electrolyte [26]. Although in the description of energy states in electrolytes the Boltzmann distribution law is relevant (opposite to Fermi-Dirac distribution that is valid for metals), it is common to consider the "Fermi" level of the electrolyte [30].

After the introduction of the most important quantities, it is important to understand why the work function changes during the adsorption of hydrogen at the solid/liquid interface. When a metallic electrode and an electrolyte are put together,
Fig. 2 Energy levels at metal/ electrolyte interface in electronic equilibrium. Indicated quantities are defined in the text

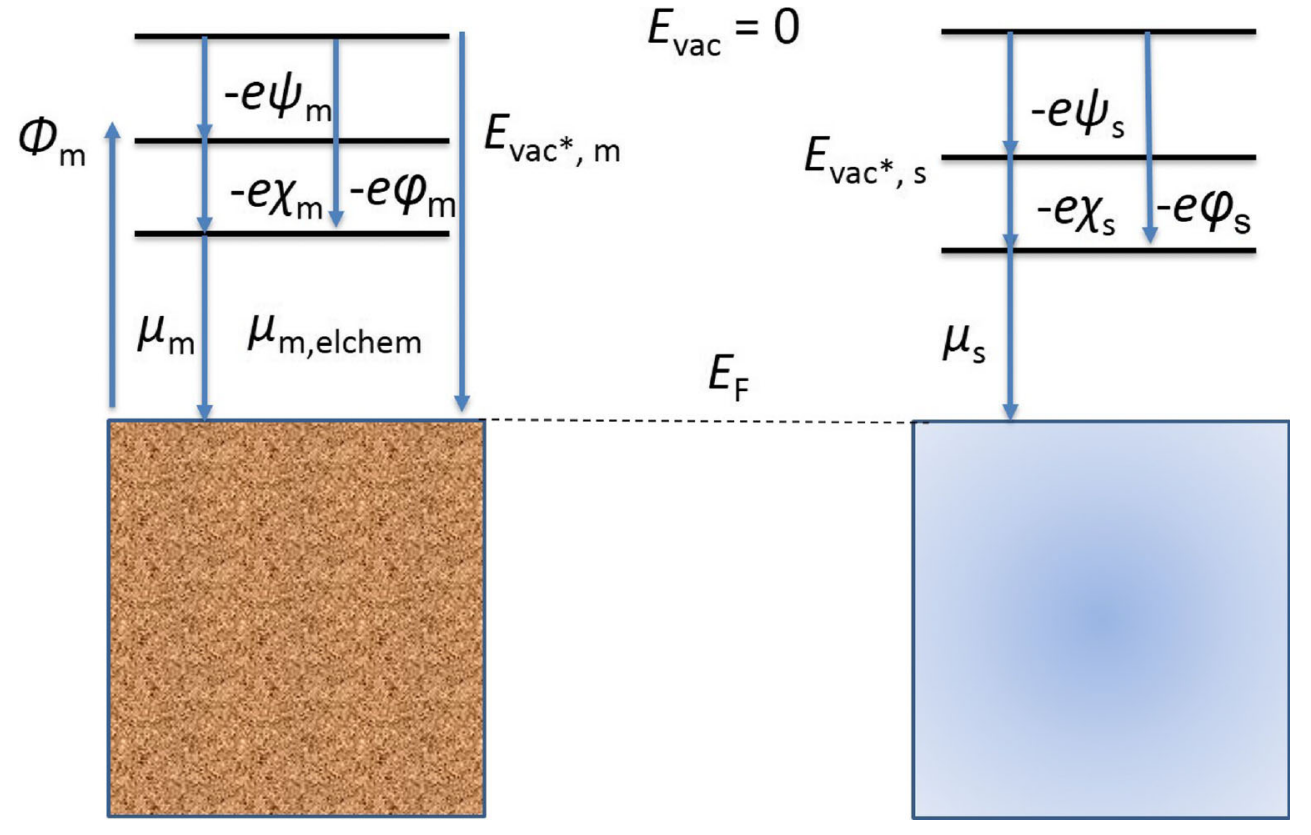


they almost instantaneously establish an electronic equilibrium (their electrochemical potentials must be equal). Due to the fact that electrons at the Fermi level in the metal and at the Fermi level in the electrolyte (redox potential) are different, the electronic equilibrium will be assured by the redistribution of the charge at the interface and by establishing the Galvani potential difference between metal and electrolyte $\left(\Delta \varphi_{\mathrm{m}, \mathrm{s}}\right)$.

This can be easily observed in Fig. 2 and expressed additionally with Eq. 1.

$\Delta \mu_{\mathrm{m}, \mathrm{s}}=e \Delta \varphi_{\mathrm{s}, \mathrm{m}}$

The working principle of SKP is based on measuring the Volta potential difference usually at the following: (1) two or more different locations of the solid surface, (2) two or more different solid materials that are in contact, (3) at the interface between solid and liquid. In our case, when the electrode and the electrolyte come in contact, their Fermi levels equilibrate. In the electronic equilibrium, due to the difference in the work functions of the metal and its analogue in the electrolyte $\left(\Delta \Phi_{\mathrm{m}, \mathrm{s}}\right)$, the Volta potential difference $\left(\Delta \psi_{\mathrm{m}, \mathrm{s}}\right)$ will be established [23] as shown in Fig. 2, described additionally with Eq. 2.

$\Delta \Phi_{\mathrm{m}, \mathrm{s}}=\mathrm{e} \Delta \psi_{\mathrm{s}, \mathrm{m}}$

If the chemical potential of the electrons in the metal and the redox potential of the electrolyte do not change, then the Galvani potential difference must remain constant (Eq. 2). If the Galvani potential difference remains constant during the adsorption of hydrogen, then any change in the Volta potential difference will be equivalent to the change in the surface potential difference at the metal/electrolyte interface $\left(\Delta \chi_{\mathrm{m}, \mathrm{s}}\right)$ and vice versa. This should be evident from Eq. 3 .

$\Delta \varphi_{\mathrm{m}, \mathrm{s}}=\Delta \psi_{\mathrm{m}, \mathrm{s}}+\Delta \chi_{\mathrm{m}, \mathrm{s}}$

At the same time, the Galvani potential difference may be written as the sum of a contribution arising from the charge separation across the interface $\left(g(\text { ion })_{\mathrm{m}, \mathrm{s}}\right)$ and one arising from the dipole $\left(g(\operatorname{dip})_{\mathrm{m}, \mathrm{s}}\right)$ either due to the water orientation or from the dipole created by specific adsorption (e.g., hydrogen), as shown with Eq. 4.

$\Delta \varphi_{\mathrm{m}, \mathrm{s}}=g(\text { ion })_{\mathrm{m}, \mathrm{s}}+g(\operatorname{dip})_{\mathrm{m}, \mathrm{s}}$

Existing dipoles at the interface are contributors to the surface potential of the metal in the presence of the electrolyte $\left(g(\mathrm{dip})_{\mathrm{m},(\mathrm{s})}\right)$, where $\delta \chi_{\mathrm{m}}$, (s) is a change of the surface potential of metal in presence of the electrolyte (Eq. 5):

$g(\operatorname{dip})_{\mathrm{m},(\mathrm{s})}=\chi_{\mathrm{m}+} \delta \chi_{\mathrm{m},(\mathrm{s})}$

or existing dipoles at the interface are contributors to the surface potential of the electrolyte in the presence of the metal $\left(g(\operatorname{dip})_{\mathrm{s},(\mathrm{m})}\right)$, where $\delta \chi_{\mathrm{s},(\mathrm{m})}$ is a change of the surface potential of electrolyte in presence of the metal (Eq. 6):

$g(\operatorname{dip})_{\mathrm{s},(\mathrm{m})}=\chi_{\mathrm{s}}+\delta \chi_{\mathrm{s},(\mathrm{m})}$

The total potential drop at the interface related to dipoles is given as follows:

$g(\operatorname{dip})_{\mathrm{m}, \mathrm{s}}=g(\operatorname{dip})_{\mathrm{m},(\mathrm{s})}-g(\operatorname{dip})_{\mathrm{s},(\mathrm{m})}$

From Eqs. 3-7, the link between the Volta potential difference and changes in surface potentials at metal/electrolyte interface is given by Eq. 8 .

$\Delta \psi_{\mathrm{m}, \mathrm{s}}=\delta \chi_{\mathrm{m},(\mathrm{s})}-\delta \chi_{\mathrm{s},(\mathrm{m})}+g($ ion $)$

This means that the adsorption of a proton from the electrolyte at the metallic surface will introduce $\mathrm{M}-\mathrm{H}$ dipoles at the interface, alter the potential drop due to existing water dipoles having increased probability to be oriented with hydrogen towards the metallic surface, and potentially alter the existing charge separation at the interface due to the enhanced concentration of protons in the interfacial region due to the process of proton adsorption/desorption. So, practically measuring the Volta potential difference, we measure the change in the work function of both phases equivalent to the change in surface potentials of both phases due to the adsorption of hydrogen. Important to notice is that Eq. 8 reflects the total change in the electrostatic energy at the interface due to hydrogen adsorption. Similar to the explained methodology would be to adsorb molecular hydrogen at the metal surface covered by a thin water layer or ideally covered by a water bilayer that responds to the dimensions of the electric double layer. In that case, the Volta potential difference should be measured before and after hydrogen adsorption. Similar investigations were initiated in the groups of Ertl [31] and Weaver [32]. Ertl's work was based on adsorption at gas/solid interface, without water layer, while the work of Weaver did include water layer. Both of them investigated the change in the work function upon adsorption; however, while Ertl did not analyze adsorption through water layer, Weaver had a too complex system $(\operatorname{Pt}(111)$ surface modified with different doses of $\mathrm{K}^{+}$cation, exposed to different amounts of solvent and different doses of $\mathrm{CO}$ ) to avoid some serious artifacts, including unclear causes of change in the work function. Importantly, none of them analyzed a series of metals.

\section{The importance of Pauling equation}

To solidify our approach, it is worth to recall one of the earliest models describing indirectly the connection between the work function and the adsorption energy, namely the Pauling equation: 


$$
\begin{aligned}
E(\mathrm{M}-\mathrm{H})= & 0.5\{D(\mathrm{M}-\mathrm{M})+D(\mathrm{H}-\mathrm{H})\} \\
& +23.06\left(X_{\mathrm{M}}-X_{\mathrm{H}}\right)^{2}
\end{aligned}
$$

where $E(\mathrm{M}-\mathrm{H})$ is the energy of the metal-hydrogen bond strength; $D(\mathrm{M}-\mathrm{M})$ - the dissociation energy of the $\mathrm{M}-\mathrm{M}$ bond in the metal lattice, usually obtained from the sublimation energy; $D(\mathrm{H}-\mathrm{H})$ - the dissociation energy of the hydrogen molecule; $X_{\mathrm{M}}$ - the electronegativity of the metal; and $X_{\mathrm{H}}$-the electronegativity of the hydrogen atom. The link between adsorption enthalpy and bond strength is given as follows (Eq. 10):

$E(\mathrm{M}-\mathrm{H})=0.5 D(\mathrm{H}-\mathrm{H})-\Delta H_{\text {ads }}(\mathrm{M}-\mathrm{H})$

where $\Delta H_{\mathrm{ads}}(\mathrm{M}-\mathrm{H})$ is the enthalpy of adsorption of hydrogen. Considering that only d-metals (transition metals) exhibit significant electrocatalytic activity for HER and only d-metals exhibit measurable coverage with hydrogen at the reversible potential of HER, further analysis will be focused only on them. In the initial analysis of Eq. 9, by Eley [33] and Rüetschi and Delahay [34], they disregarded the electronegativity term. In their opinion, the difference in electronegativity between various metals and hydrogen is negligible. This resulted in an inter-dependence where increase in work function is coupled with an increase in the $\mathrm{M}-\mathrm{H}$ bond strength. However, Bockris and Conway [35], and later Trasatti [6], emphasized that the electronegativity term is of essential importance. In their case, an increase in the work function was coupled with a drop in the $\mathrm{M}-\mathrm{H}$ bond strength. The dependence is shown in Fig. 3. It seems that for the early transition metals where the difference in the electronegativity between the metal and the hydrogen atom is the largest, the $\mathrm{M}-\mathrm{H}$ bond is the strongest. With the increase in the number of d-electrons in the valence level, the difference in electronegativity

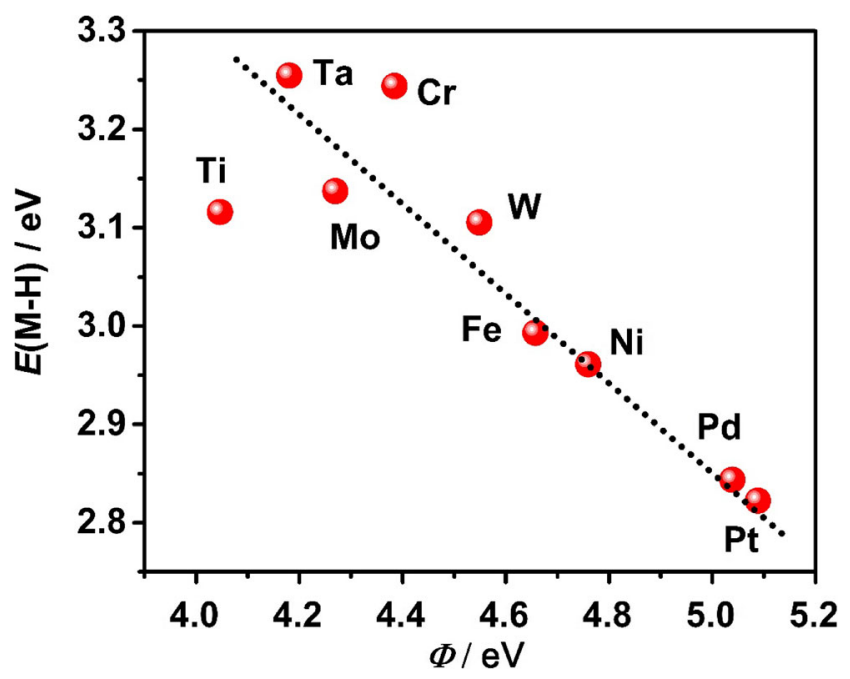

Fig. 3 Relationship between $\mathrm{M}-\mathrm{H}$ bond strength and work function, for d-metals. Data are extracted from the Fig. 5 in [6]. Replotted relation originates from [21]. Published by the PCCP Owner Societies approaches to zero, indicating that the $\mathrm{M}-\mathrm{H}$ bond becomes weaker.

From Fig. 3, it is straightforward that in a case of d-metals, the adsorption of hydrogen should cause a drop in the work function. The stronger the $\mathrm{M}-\mathrm{H}$ bond is, the larger will be the drop in the work function. What can be noticed from Pauling equation and Eq. 10 is that the adsorption energy of the $\mathrm{M}-\mathrm{H}$ bond formation can be brought in direct relation to the change in the electrostatic energy at the interface during hydrogen adsorption (Eq. 8). As specified above, the entire analysis is for d-metals. This is important to emphasize because Trasatti [6] showed that sp-metals behave opposite to d-metals, meaning that the work function increases with the strength of hydrogen adsorption. He attributed that to interfacial water structure and orientation, although it can be also due to different kinds of adsorption site. If adsorption is on top, having in mind the direction of $\mathrm{M}-\mathrm{H}$ dipole, we would expect an increase in the work function; however, if the adsorption site is an interatomic hollow site, then the dipole is oriented in a way that causes a drop in the work function. We previously addressed controversies on exact location of hydrogen adsorption in the critical review on hydrogen electrocatalysis [18]. The approach introduced in this work brings also opportunity to study different crystal facets and the behavior of water on various surfaces, amongst others, which could additionally help to understand polycrystalline materials.

\section{Experimental details and preliminary results}

Summarizing the theoretical aspects of the methodology, we can say the following:

(1) From Fig. 1, we conclude qualitatively that the $\mathrm{M}-\mathrm{H}$ bond formation should alter the work function of the metal.

(2) A change in the work function will be manifested through the Volta potential change, which can be measured by SKP.

(3) For a system where chemical potentials of the metal and the electrolyte do not change, the Galvani potential difference has to remain constant. This means that if the work function is changing due to the $\mathrm{M}-\mathrm{H}$ bond formation, the change in work function originates strictly in the change of the surface potential, which has to be equal to the change in the Volta potential (which is measurable). This can be seen easily from Fig. 2.

(4) Equation 8 illustrates what we measure first at the metal/ electrolyte interface and afterwards at the same interface after the adsorption of hydrogen. That is the total change in the electrostatic energy at the interface due to the $\mathrm{M}-\mathrm{H}$ bond formation including the following: the change in surface potential difference of the metal/electrolyte 
interface due to hydrogen adsorption plus the free charge (ions) introduced into the double layer due to the hydrogen adsorption/desorption. So, if classical adsorption energies are a thermal consequence of the $\mathrm{M}-\mathrm{H}$ bond formation, we measure the total electrostatic consequence of the $\mathrm{M}-\mathrm{H}$ bond formation.

(5) From the dependence shown in Fig. 3, it is straightforward that the larger the difference in electronegativity between metal and hydrogen, the stronger the $\mathrm{M}-\mathrm{H}$ bond and consequently the larger the drop in the work function. Interestingly, the slope of dependence in Fig. 3 corresponds to the slope between the change in the work function and the potential difference that is a driving force for underpotential deposition of metals, introduced by Gerischer et al. [36]. If the work function increases for $1 \mathrm{eV}$, the bond strength decreases for $0.5 \mathrm{eV}$. The exact proportionality between the change in work function and the adsorption energy depends on the adsorbate coverage. If lateral interactions are of relevance (usually at higher coverages), then the interaction parameter can enhance or locally reduce the $\mathrm{H}_{\mathrm{ad}}$ adsorption energy, consequently altering the local $\mathrm{M}-\mathrm{H}$ bond strength as well as the value of the $\mathrm{M}-\mathrm{H}$ dipole (or even sign) that is manifested by a change in the local work function. It is indeed essential to know the quantitative relation between work function and coverage. However, we operate at conditions of low dosage of hydrogen so the coverage is expected to be rather low, including the absence of lateral interactions. Under given conditions, a metal that has intrinsically high tendency to bind hydrogen strongly (strong dipole) will have at the same time higher coverage, which will be detected by larger drop in work function than for the metal that has intrinsically tendency to bind hydrogen weakly (weak dipole and low coverage). Therefore, we believe that the trends shown in Fig. 4 are relevant. Important to emphasize is that observed trend could be distorted due to appearance of $\mathrm{H}_{\mathrm{upd}}$ (underpotentially deposited hydrogen). Our initial point of view was that under neutral $\mathrm{pH}$ conditions of our experiment (hydrogen introduced through the thin water layer) $\mathrm{H}_{\text {upd }}$ is not significant as shown in the work of Qiao et al. [37]. However, on the contrary, under neutral $\mathrm{pH}$ conditions and very clean experimental conditions, $\mathrm{H}_{\text {upd }}$ exists on platinum [38], implying that under our measurement conditions, $\mathrm{H}_{\text {upd }}$ exists and it could interfere with the adsorption of atomic hydrogen originating from the hydrogen gas. This is a very important point indicating why our assumption about a negligible interaction parameter and its impact on coverage could be challenged. At the same time, we are aware that $\mathrm{H}_{\text {upd }}$ exists only on noble metals and, despite probably not being an intermediate, it plays a special role in the activity of HER that was never really resolved. Understanding the role of $\mathrm{H}_{\text {upd }}$ would require to find a link between $\mathrm{H}_{\text {upd }}$ and $\mathrm{H}_{\mathrm{ad}}$ that is an intermediate sometimes called overpotentially deposited hydrogen or $\mathrm{H}_{\mathrm{opd}}[18]$.

Preliminary experiments using the SKP setup with an atmosphere and humidity control were done on a series of seven noble metals ( $\mathrm{Au}, \mathrm{Pd}, \mathrm{Pt}, \mathrm{Cu}, \mathrm{Rh}, \mathrm{Ir}, \mathrm{Ru})$ [21]. Before experiment, the samples (polycrystalline metals $5 \mathrm{~mm}$ diameter disk from MaTeck, Jülich, Germany) were polished with 1 and $0.3 \mathrm{~mm}$ alumina suspensions on a polishing cloth (Struers, MD Mol) followed by a short sonication in water, extensive washing in ultrapure water (PureLab Plus system, Elga, 18 $\mathrm{MV}, \mathrm{TOC}, 3 \mathrm{ppb}$ ) and drying in a flow of argon. In this way, the influence of formed surface oxides, organic impurities, etc. is minimized. All samples were fixed from the bottom with conductive tape to a metallic holder. Due to possible influence of impurities from the commercial electrolytes, experiments were executed by covering the metallic samples with thin water layer through which gases were adsorbed on metallic surfaces. SKP tip was a CrNi alloy with diameter of $500 \mu \mathrm{m}$ and a known work function. Prior to the measurements, the SKP tip was calibrated using a $\mathrm{Cu} / \mathrm{CuSO} 4$ (sat.) electrochemical couple. The samples were transferred inside the measurement chamber, which was initially under a nitrogen atmosphere and low relative humidity $(1 \% \mathrm{RH}$, referred in the following as dry condition). After achieving steady atmospheric and humidity conditions, the measurement was started. The change in work function was measured continuously on an area of $500 \mathrm{~mm} \times 500 \mathrm{~mm}$, giving an array of 100 measurement points per sample. The average value of work function is taken out of these 100 points and introduced in Fig. $4 \mathrm{a}$ in section $\mathrm{N}_{2}$ "dry." The same measurement was repeated for each metal. In the second step, the humidity of $95-98 \%$ was set in a nitrogen atmosphere. Again, the average value of work function is introduced in Fig. $4 \mathrm{a}$ as $\mathrm{N}_{2}$ "humid." In the third step, again under conditions of a maximum humidity of $95-98 \%$, the measurement was performed under a hydrogen atmosphere by using 5\% hydrogen in nitrogen (so-called forming gas). The obtained average value of the work function is introduced in section $\mathrm{H}_{2}$ humid as shown in Fig. 4a.

The change in the work function from nitrogen dry conditions to nitrogen humid conditions was minor. This is due to the fact that at dry conditions, we have already a thin water layer that can be removed only in vacuum. After the introduction of hydrogen through the water layer, the work function dropped significantly ( $\Delta \Phi$ shown in Fig. $4 \mathrm{a}$, between $\mathrm{N}_{2}$ humid and $\mathrm{H}_{2}$ humid). This is an indicator that the $\mathrm{M}-\mathrm{H}$ bond creates a dipole that reduces the work function of the metal. Here it should be mentioned that the M-H dipole could partly include ionic character, depending on the difference in the electronegativity between hydrogen and metal. For metals which have intermediate $\mathrm{M}-\mathrm{H}$ bond strengths, the desorption 


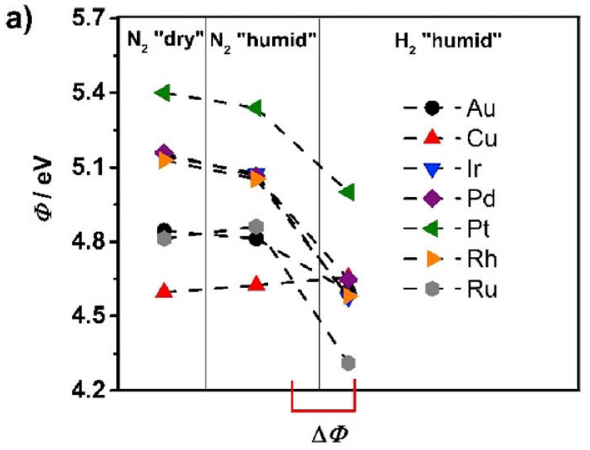

Fig. 4 a Change in the work function of a group of metals under various atmospheres measured by SKP at room temperature. After achieving steady atmospheric and humidity conditions, an area of $500 \mathrm{~mm} \times$ $500 \mathrm{~mm}$ was measured, giving an array of 100 measurement points per sample. b Experimental "volcano" plot where the exchange current for

of protons into the water layer is probable, implying that a hydrogen electrode is formed [39, 40]. Importantly, the experiment confirms that a change in the work function upon adsorption of hydrogen through the water layer is a relevant interfacial parameter directly related to the adsorption energy. The obtained values can be influenced also by impurities in the gases. In the existing setup, an oxygen sensor was installed; however, impurities due to hydrocarbons traces in the hydrogen or the $\mathrm{CO}_{2}$ that can form carbonates with water and impurify metal surfaces cannot be excluded. In the future work, this will be carefully investigated by means of gas chromatography. It is important to keep in mind that the purpose of this initial work was to get meaningful trends and not necessarily to obtain rigidly accurate values. Despite that promoters of rigid purification protocols never showed activity trends that would contradict the trends reported by Trasatti [6] still, cleaning procedure on our future work will be complemented with electrochemical activation to be sure that the influence of impurities is definitely negligible [41]. Importantly, after the third step $\left(\mathrm{H}_{2}\right.$ humid conditions), the original value of the work function cannot be completely recovered. It seems that a fraction of the gas remains adsorbed on the surface of the wet metal even after several hours of the initial atmosphere $\left(\mathrm{N}_{2}\right.$ dry conditions). This should not be surprising since the desorption of hydrogen depends on the $\mathrm{M}-\mathrm{H}$ bond strength that in the case of some metals is substantial.

Finally, from Fig. 4b, it seems that the well-established experimental values of exchange current densities for HER can be correlated with a change in the work function upon adsorption of hydrogen through the water layer in a form of a "volcano" curve. Given dependence complies with one of the implicit goals of our research and that is to give wellgrounded answer to whether Sabatier principle in electrocatalysis is valid or not, taking into consideration that for different metals, different rate determining steps can control the reaction. Despite of the substantial criticism that exists towards the concept of volcano curves [19] and the view that

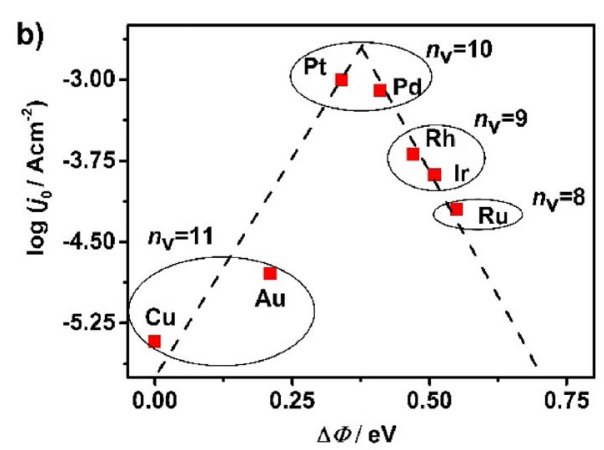

HER (values taken from [6]) is related to the experimentally determined change in the work function of the metal upon adsorption of hydrogen through the water layer. For various metals, the number of valence electrons $\left(n_{\mathrm{v}}\right)$ is indicated. Both plots were constructed using data from [21]. Published by the PCCP Owner Societies

thermochemistry of adsorption is insufficient to be the only foundation for theory of electrocatalysis [18], still obtained activity trends and anticipated factors that limit electrocatalytic activity are valid. This fact holds, surprisingly, independently from conditions at which this analysis is conducted. Overlap in the trends in adsorption energies between gasphase data and overpotential-dependent electrochemical data [6] is surprising and suggests that adsorption energies acquired at equilibrium conditions could be relevant and share some additional light on hydrogen electrocatalysis. The dependence we obtained is similar to the previous relations where the exchange currents were correlated to the adsorption energies $[6,16]$. However, the conditions in our experiment are different from the conditions at which electrochemical adsorption heats are extracted [6] as well as from conditions at which $\mathrm{H}_{\text {upd }}$ appears at the electrode surface, as explained above. Our values originate from the gas-phase hydrogen that was adsorbed at equilibrium conditions through the ultra-thin water layer. This is identical to Tafel reaction during HER or hydrogen oxidation reaction (HOR). Importantly, the trend in work function change we obtained is a function of the valence of electrons in a way that is expected. Namely, the larger the number of valence electrons, the weaker will be the $\mathrm{M}-\mathrm{H}$ bond strength. Considering that the observed change in the work function is equal to the change in the Volta potential difference after hydrogen is adsorbed through the water layer (Eq. 8) and that it is equivalent to the total change in the electrostatic energy due to process of $\mathrm{M}-\mathrm{H}$ formation, we can name these values experimental adsorption energies. It seems that metals with 10 valence electrons $\left(n_{\mathrm{v}}\right)$ like Pt and Pd have the optimal experimental adsorption energies, exhibiting also the highest exchange current densities. It is interesting that the experimental adsorption energy for Pt is around $-0.35 \mathrm{eV}$, similar to the value obtained by DFT on Pt model surfaces [16]. At the same time, the experimental volcano shown in Fig. 4 indicates that $\mathrm{H}$ adsorption on $\mathrm{Pt}$ is bonded slightly weaker than the optimal, opposite to the 
conventional computational model [16]. From the preliminary results, it seems that the proposed methodology treats the metal/electrolyte interface in a very meaningful manner [42, 43]. At the same time, it opens a perspective for comparative computational vs. experimental studies [44] as well as for comparative thermal catalysis $[45,46]$ vs. electrocatalysis studies $[24,47]$ with the goal of establishing unifying concepts in catalysis [48].

\section{Conclusions}

We propose an experimental methodology for quantitative description of adsorption energies at solid/liquid interfaces. In this way, for important molecules, like hydrogen and oxygen, the strength of the bond which they establish with the catalyst surface can be probed. This is of major significance for electrocatalytic reactions, because the adsorption of species on polycrystalline materials will be now experimentally accessible, for which DFT exhibits limitations. The current research was conducted at open circuit conditions; however, estimating adsorption energies under conditions of controlled electrode potential or current is the next logical step. In the future, detailed experimental trends in adsorption energies can be established for classes of chemical compounds and materials (d-metals, sp-metals, alloys, oxides, carbides, borides, metal-nitrogen-carbon composites, etc.), which are of interest in heterogenous catalysis as gas-evolving or gas-consuming electrodes.

Acknowledgments The authors express their gratitude to Prof. Alexander Auer for interesting discussion and useful comments that improved the quality of this work.

Funding Open Access funding provided by Projekt DEAL.

Open Access This article is licensed under a Creative Commons Attribution 4.0 International License, which permits use, sharing, adaptation, distribution and reproduction in any medium or format, as long as you give appropriate credit to the original author(s) and the source, provide a link to the Creative Commons licence, and indicate if changes were made. The images or other third party material in this article are included in the article's Creative Commons licence, unless indicated otherwise in a credit line to the material. If material is not included in the article's Creative Commons licence and your intended use is not permitted by statutory regulation or exceeds the permitted use, you will need to obtain permission directly from the copyright holder. To view a copy of this licence, visit http://creativecommons.org/licenses/by/4.0/.

\section{References}

1. Yeager E (1966) Challenging problems in electrochemistry. J Electrochem Soc 113(7):153C. https://doi.org/10.1149/1.2424078

2. Zagal JH, Koper MTM (2016) Reactivity descriptors for the activity of molecular MN4 catalysts for the oxygen reduction reaction.
Angew Chem Int Ed 55(47):14510-14521. https://doi.org/10.1002/ anie. 201604311

3. Appleby AJ, Zagal JH (2011) Free energy relationships in electrochemistry: a history that started in 1935. J Solid State Electrochem 15(7-8):1811-1832. https://doi.org/10.1007/s10008-011-1394-8

4. Bowden FP, Rideal EK (1928) The electrolytic behaviour of thin films. Part I. Hydrogen. Proc R Soc Math Phys Eng Sci 120(784): 59-79. https://doi.org/10.1098/rspa.1928.0135

5. Parsons R (1958) The rate of electrolytic hydrogen evolution and the heat of adsorption of hydrogen. Trans Faraday Soc 54:1053. https://doi.org/10.1039/tf9585401053

6. Trasatti S (1972) Work function, electronegativity, and electrochemical behaviour of metals III. Electrolytic hydrogen evolution in acid solutions. J Electroanal Chem Interfacial Electrochem 39: 163-184. https://doi.org/10.1016/S0022-0728(72)80485-6

7. Bockris JO, Otagawa T (1983) Mechanism of oxygen evolution on perovskites. J Phys Chem 87(15):2960-2971. https://doi.org/10. 1021/j100238a048

8. Bockris JO (1984) The electrocatalysis of oxygen evolution on perovskites. J Electrochem Soc 131(2):290. https://doi.org/10. $1149 / 1.2115565$

9. Kunimatsu K, Senzaki T, Samjeské G, Tsushima M, Osawa M (2007) Hydrogen adsorption and hydrogen evolution reaction on a polycrystalline Pt electrode studied by surface-enhanced infrared absorption spectroscopy. Electrochim Acta 52(18):5715-5724. https://doi.org/10.1016/j.electacta.2006.12.007

10. Stamenkovic V, Mun BS, Mayrhofer KJJ, Ross PN, Markovic NM, Rossmeisl J, Greeley J, Nørskov JK (2006) Changing the activity of electrocatalysts for oxygen reduction by tuning the surface electronic structure. Angew Chem Int Ed 45(18):2897-2901. https://doi. org/10.1002/anie.200504386

11. Koper MTM (2013) Analysis of electrocatalytic reaction schemes: distinction between rate-determining and potential-determining steps. J Solid State Electrochem 17(2):339-344. https://doi.org/10. 1007/s10008-012-1918-x

12. Balandin AA (1962) The multiplet theory of catalysis. Structural factors in catalysis. Russ Chem Rev 31(11):589-614. https://doi. org/10.1070/RC1962v031n11ABEH001323

13. Koper MTM (2016) Activity volcanoes for the electrocatalysis of homolytic and heterolytic hydrogen evolution. J Solid State Electrochem 20(4):895-899. https://doi.org/10.1007/s10008-0153036-Z

14. Koper MTM (2011) Thermodynamic theory of multi-electron transfer reactions: implications for electrocatalysis. J Electroanal Chem 660(2):254-260. https://doi.org/10.1016/j.jelechem.2010. 10.004

15. Nørskov JK, Rossmeisl J, Logadottir A, Lindqvist L, Kitchin JR, Bligaard T, Jónsson H (2004) Origin of the overpotential for oxygen reduction at a fuel-cell cathode. J Phys Chem B 108(46): 17886-17892. https://doi.org/10.1021/jp047349j

16. Nørskov JK, Bligaard T, Logadottir A, Kitchin JR, Chen JG, Pandelov S, Stimming U (2005) Trends in the exchange current for hydrogen evolution. J Electrochem Soc 152(3):J23. https://doi. org/10.1149/1.1856988

17. Schmickler W, Trasatti S (2006) Comment on "Trends in the exchange current for hydrogen evolution" [J. Electrochem. Soc., 152, J23 (2005)]. J Electrochem Soc 153(12):L31. https://doi.org/10. $1149 / 1.2358294$

18. Zeradjanin AR, Grote J-P, Polymeros G, Mayrhofer KJJ (2016) A critical review on hydrogen evolution electrocatalysis: re-exploring the volcano-relationship. Electroanalysis 28(10):2256-2269. https://doi.org/10.1002/elan.201600270

19. Quaino P, Juarez F, Santos E, Schmickler W (2014) Volcano plots in hydrogen electrocatalysis - uses and abuses. Beilstein $\mathrm{J}$ Nanotechnol 5:846-854. https://doi.org/10.3762/bjnano.5.96 
20. Santos E, Quaino P, Schmickler W (2012) Theory of electrocatalysis: hydrogen evolution and more. Phys Chem Chem Phys 14(32):11224-11233. https://doi.org/10.1039/c2cp40717e

21. Zeradjanin AR, Vimalanandan A, Polymeros G, Topalov AA, Mayrhofer KJJ, Rohwerder M (2017) Balanced work function as a driver for facile hydrogen evolution reaction - comprehension and experimental assessment of interfacial catalytic descriptor. Phys Chem Chem Phys 19(26):17019-17027. https://doi.org/10. 1039/C7CP03081A

22. Zeradjanin AR (2018) Is a major breakthrough in the oxygen electrocatalysis possible? Curr Opin Electrochem 9:214-223. https://doi.org/10.1016/j.coelec.2018.04.006

23. Rohwerder M, Turcu F (2007) High-resolution Kelvin probe microscopy in corrosion science: scanning Kelvin probe force microscopy (SKPFM) versus classical scanning Kelvin probe (SKP). Electrochim Acta 53(2):290-299. https://doi.org/10.1016/j. electacta.2007.03.016

24. Zeradjanin AR, Polymeros G, Toparli C, Ledendecker M, Hodnik N, Erbe A, Rohwerder M, la Mantia F (2020) What is the trigger for the hydrogen evolution reaction? - towards electrocatalysis beyond the Sabatier principle. Phys Chem Chem Phys 22(16):8768-8780. https://doi.org/10.1039/D0CP01108H

25. Trasatti S (1986) The absolute electrode potential: an explanatory note (Recommendations 1986). J Electroanal Chem Interfacial Electrochem 209(2):417-428. https://doi.org/10.1016/00220728(86)80570-8

26. Trasatti S, Parsons R (1986) Interphases in systems of conducting phases. J Electroanal Chem Interfacial Electrochem 205(1-2):359376. https://doi.org/10.1016/0022-0728(86)90251-2

27. Frumkin AN, Petrii OA (1975) Potentials of zero total and zero free charge of platinum group metals. Electrochim Acta 20(5):347-359. https://doi.org/10.1016/0013-4686(75)90017-1

28. Iwasita T, Xia X (1996) Adsorption of water at Pt(111) electrode in $\mathrm{HClO} 4$ solutions. The potential of zero charge. J Electroanal Chem 411(1-2):95-102. https://doi.org/10.1016/0022-0728(96)04576-7

29. Thiel PA, Madey TE (1987) The interaction of water with solid surfaces: fundamental aspects. Surf Sci Rep 7(6-8):211-385. https://doi.org/10.1016/0167-5729(87)90001-X

30. Khan SUM, Kainthla RC, Bockris JOM (1987) The redox potential and the Fermi level in solution. J Phys Chem 91(23):5974-5977. https://doi.org/10.1021/j100307a032

31. Christmann K, Ertl G, Pignet T (1976) Adsorption of hydrogen on a Pt(111) surface. Surf Sci 54(2):365-392. https://doi.org/10.1016/ 0039-6028(76)90232-6

32. Villegas I, Kizhakevariam N, Weaver MJ (1995) Infrared spectroscopy of model electrochemical interfaces in ultrahigh vacuum: some implications for ionic and chemisorbate solvation at electrode surfaces. Surf Sci 335:300-314. https://doi.org/10.1016/00396028(95)00448-3

33. Eley DD (1951) Molecular hydrogen and metallic surfaces. J Phys Chem 55(6):1017-1036. https://doi.org/10.1021/j150489a022

34. Rüetschi P, Delahay P (1955) Hydrogen overvoltage and electrode material. A theoretical analysis. J Chem Phys 23(1):195-199. https://doi.org/10.1063/1.1740527

35. Conway BE, Bockris JO (1957) Electrolytic hydrogen evolution kinetics and its relation to the electronic and adsorptive properties of the metal. J Chem Phys 26(3):532-541. https://doi.org/10.1063/ 1.1743339

36. Kolb DM, Przasnyski M, Gerischer H (1974) Underpotential deposition of metals and work function differences. J Electroanal Chem Interfacial Electrochem 54(1):25-38. https://doi.org/10.1016/ S0022-0728(74)80377-3

37. Wang X, Xu C, Jaroniec M, Zheng Y, Qiao SZ (2019) Anomalous hydrogen evolution behavior in high-pH environment induced by locally generated hydronium ions. Nat Commun 10(1):4876. https://doi.org/10.1038/s41467-019-12773-7

38. Climent MA, Valls MJ, Feliu JM, Aldaz A, Clavilier J (1992) The behaviour of platinum single-crystal electrodes in neutral phosphate buffered solutions. J Electroanal Chem 326(1-2):113-127. https:// doi.org/10.1016/0022-0728(92)80507-Z

39. Evers S, Senöz C, Rohwerder M (2013) Hydrogen detection in metals: a review and introduction of a Kelvin probe approach. Sci Technol Adv Mater 14(1):014201. https://doi.org/10.1088/14686996/14/1/014201

40. Evers S, Rohwerder M (2012) The hydrogen electrode in the "dry": a Kelvin probe approach to measuring hydrogen in metals. Electrochem Commun 24:85-88. https://doi.org/10.1016/j.elecom. 2012.08.019

41. Climent V, Feliu JM (2011) Thirty years of platinum single crystal electrochemistry. J Solid State Electrochem 15(7-8):1297-1315. https://doi.org/10.1007/s10008-011-1372-1

42. Dubouis N, Grimaud A (2019) The hydrogen evolution reaction: from material to interfacial descriptors. Chem Sci 10(40):91659181. https://doi.org/10.1039/C9SC03831K

43. Zhang R, Pearce PE, Duan Y, Dubouis N, Marchandier T, Grimaud A (2019) Importance of water structure and catalyst-electrolyte interface on the design of water splitting catalysts. Chem Mater 31(20):8248-8259. https://doi.org/10.1021/acs.chemmater. $9 \mathrm{~b} 02318$

44. Exner KS (2020) Comparison of the conventional volcano analysis with a unifying approach: material screening based on a combination of experiment and theory. J Phys Chem C 124(1):822-828. https://doi.org/10.1021/acs.jpcc.9b10860

45. Schlögl R (2016) Selective oxidation: from a still immature technology to the roots of catalysis science. Top Catal 59(17-18):14611476. https://doi.org/10.1007/s11244-016-0684-x

46. Schlögl R (2015) Heterogeneous catalysis. Angew Chem Int Ed 54(11):3465-3520. https://doi.org/10.1002/anie.201410738

47. Zeradjanin AR, Menzel N, Strasser P, Schuhmann W (2012) Role of water in the chlorine evolution reaction at $\mathrm{RuO} 2$-based electrodes-understanding electrocatalysis as a resonance phenomenon. ChemSusChem 5(10):1897-1904. https://doi.org/10.1002/cssc. 201200193

48. Maganas D, Trunschke A, Schlögl R, Neese F (2016) A unified view on heterogeneous and homogeneous catalysts through a combination of spectroscopy and quantum chemistry. Faraday Discuss 188:181-197. https://doi.org/10.1039/C5FD00193E

Publisher's note Springer Nature remains neutral with regard to jurisdictional claims in published maps and institutional affiliations. 